\title{
Improved production of fatty alcohols in cyanobacteria by metabolic engineering
}

\author{
Lun Yao ${ }^{1,2+}$, Fengxia $\mathrm{Qi}^{1,2+}$, Xiaoming Tan ${ }^{1}$ and Xuefeng $\mathrm{Lu}^{1 *}$
}

\begin{abstract}
Background: Fatty alcohols are widely used in industrial chemicals. The biosynthetic pathways for fatty alcohols are diverse and widely existing in nature. They display a high capacity to produce fatty alcohols by the metabolic engineering of different microbe hosts. Direct recycling of carbon dioxide to fatty alcohols can be achieved by introducing a fatty alcohol-producing pathway into photosynthetic cyanobacteria. According to our precious reports, a relatively low yield of fatty alcohols was obtained in the genetically engineered cyanobacterium Synechocystis sp. PCC 6803.

Results: The photosynthetic production of fatty alcohols in Synechocystis sp. PCC 6803 was improved through heterologously expressing fatty acyl-Coenzyme A (acyl-CoA) reductase gene maqu_2220 from the marine bacterium Marinobacter aquaeolei VT8. Maqu_2220 has been proved to catalyze both the four-electron reduction of fatty acyl-CoA or acyl-Acyl Carrier Protein (acyl-ACP) and the two-electron reduction of fatty aldehyde to fatty alcohol. The knockout of the aldehyde-deformylating oxygenase gene (s/l0208) efficiently blocked the hydrocarbon accumulation and redirected the carbon flux into the fatty alcohol-producing pathway. By knocking-out both s/10208 and s/10209 (encoding an acyl-ACP reductase), the productivity of fatty alcohols was further increased to $2.87 \mathrm{mg} / \mathrm{g}$ dry weight.

Conclusions: The highest yield of fatty alcohols was achieved in cyanobacteria by expressing the prokaryotic fatty acyl-CoA reductase Maqu_2220 and knocking-out the two key genes (s/l0208 and s/10209) that are involved in the alka(e)ne biosynthesis pathway. Maqu_2220 was demonstrated as a robust enzyme for producing fatty alcohols in cyanobacteria. The production of fatty alcohols could be significantly increased by blocking the hydrocarbon biosynthesis pathway.
\end{abstract}

Keywords: Fatty alcohol, Cyanobacteria, Fatty acyl-CoA reductase, Marinobacter aquaeolei VT8

\section{Background}

As concerns over the fuel shortage and environmental problems caused by the use of petroleum-based fuels, the development of biofuels has been considered as a promising choice to replace or supplement traditional fossil fuels. Numerous reviews have been published focusing on this area and various kinds of biofuels, including fatty acid-based biofuels, have been explored [1-6].

Cyanobacteria are non-food-based feedstock resources that can use non-productive land and water sources. They are able to convert solar energy and $\mathrm{CO}_{2}$ for the synthesis

\footnotetext{
* Correspondence: Ivxf@qibebt.ac.cn

${ }^{\dagger}$ Equal contributors

'Key Laboratory of Biofuels, Shandong Provincial Key Laboratory of Energy

Genetics, Qingdao Institute of Bioenergy and Bioprocess Technology,

Chinese Academy of Sciences, No. 189 Songling Road, Qingdao 266101,

China

Full list of author information is available at the end of the article
}

of co-products and do not lead to loss of ecosystems [7]. The production of biofuels and biochemicals in cyanobacteria are receiving increasing attention because of their photosynthetic capability and genetic engineering capacity. The genetic manipulation platform for cyanobacteria is well established and over 126 genomes of cyanobacterial strains have already been sequenced [8]. Several reviews have been published to illustrate the advantages and challenges in the engineering of cyanobacteria to produce valuable products $[9,10]$. Up to now, different kinds of molecules such as hydrogen, ethanol, isobutyraldehyde, isoprene, $\beta$-caryophyllene, sucrose, butanol, fatty alcohols, and fatty acids have been produced in cyanobacteria [11-21]. The activation and recycling of fatty acids in cyanobacteria has also been investigated [22,23]. In 2010, an alkane biosynthesis pathway that consists of an acyl-Acyl carrier protein reductase (AAR) and an aldehyde-deformylating oxygenase 
(ADO) has been identified in cyanobacteria [24]. A side pathway responsible for a one-step conversion of fatty aldehyde to free fatty acid by an aldehyde dehydrogenase (Orf0489) was also reported in the cyanobacterium Synechococcus elongatus PCC 7942 [25]. Its ortholog Slr0091 was also identified in the cyanobacterium strain Synechocystis sp. PCC 6803 (hereafter Synechocystis) [26].

Fatty alcohols are important industrial chemicals. They can also be good biofuel additives because of their perfect fuel properties. In nature, fatty alcohols exist as free fatty alcohols or waxes (oxygen esters of primary fatty alcohols and fatty acids) in a wide range of organisms including bacteria, plants, and mammals [27-33]. Generally, two different reaction schemes can generate primary fatty alcohols in biological systems (Figure 1A). One scheme is the four-electron reduction of fatty acyl-CoA (or acylACP) to the corresponding fatty alcohol [27]. The other scheme involves two distinct enzymes: a fatty aldehyde forming acyl-CoA reductase which catalyses a two-electron reduction of fatty acyl-CoA to a fatty aldehyde intermediate, and a fatty aldehyde reductase which catalyses the reduction of fatty aldehyde to fatty alcohol $[27,34]$. Thus, the fatty acyl-CoA (or acyl-ACP) reductases (FARs) can be divided into two general types in terms of their reaction schemes: the four-electron and two-electron reduction FARs. Additional file 1: Table S1 lists the reported FARs from different organisms as well as their substrate specificities, and types. The phylogenetic relationships of these enzymes were displayed as an unrooted tree generated by the neighbor-joining method (Additional file 2: Figure S1).

Wahlen et al. [35] characterized an enzyme (Maqu_2220) performing reduction of fatty aldehydes to fatty alcohols from Marinobacter aquaeolei VT8 (hereafter M. aquaeolei VT8). They designated Maqu_2220 a fatty aldehyde reductase (FALDR) since it could convert fatty aldehydes to fatty alcohols through an in vitro experiment [35]. Later on, Hofvander et al. performed another in vitro assay and contradictorily reported that Maqu_2220 could not only
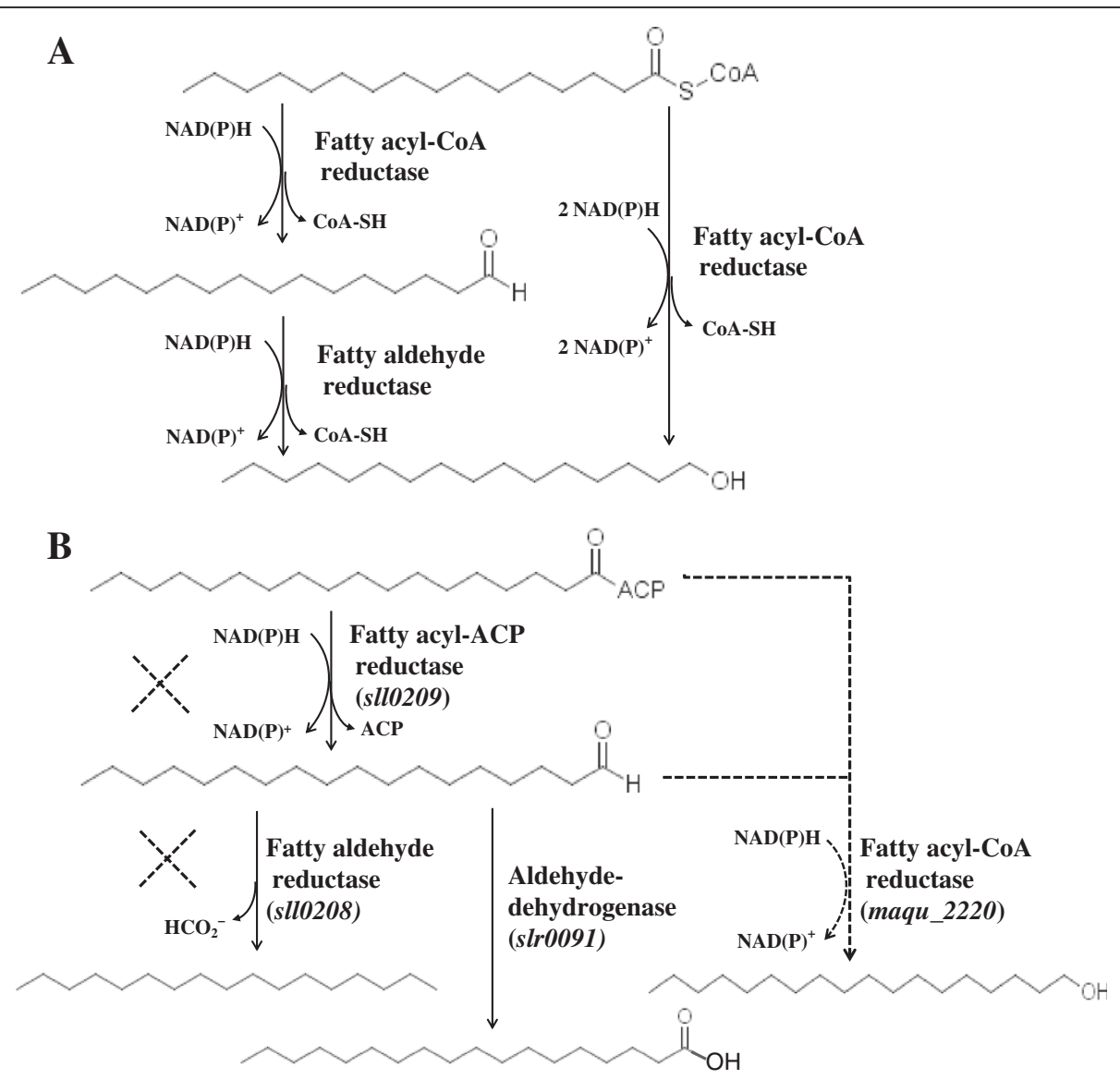

Figure 1 Fatty alcohol biosynthesis pathways. (A) Two different reaction schemes of fatty alcohol biosynthesis. Fatty alcohol could be synthesized via consecutive reduction reactions of a fatty acyl-CoA reductase and a fatty aldehyde reductase or via a single-step reduction of fatty acyl-CoA reductase. (B) Schematic view of fatty alcohol biosynthesis pathways in Synechocystis sp. PCC 6803 (Synechocystis). Solid lines show the native alka(e)ne and free fatty acid biosynthesis pathways in Synechocystis; dashed lines show the constructed fatty alcohol biosynthesis pathways in Synechocystis by knocking out the endogenous s/10208/s/10209 gene and heterologously expressing the fatty acyl-CoA reductase (maqu_2220) from Marinobacter aquaeolei VT8. 
catalyze the conversion of fatty aldehydes to fatty alcohols, but also catalyze the direct reduction of fatty acyl-CoAs (or acyl-ACPs) to fatty alcohols [36]. In vivo, however, it is still not clear which scheme is the major contributor for fatty alcohol synthesis by Maqu_2220. The gene maqu_2220 was expressed in Escherichia coli (E. coli) and a remarkable level of fatty alcohols $(1.725 \mathrm{~g} / \mathrm{L})$ were produced in an optimized mutant strain of $E$. coli under fermentation conditions [37]. In a recent paper, Saccharomyces cerevisiae was also engineered to produce fatty alcohols (almost $100 \mathrm{mg} / \mathrm{L}$ in 168 hours) by overexpressing the mouse fatty acyl-CoA reductase (mFAR1) [38].

Cyanobacteria do not have native fatty alcohol synthesis pathways. The production of fatty alcohols in cyanobacteria was investigated in our previous study by heterologously expressing the fatty acyl-CoA reductase (FAR) from mouse, jojoba (Simmondsia chinensis), and Arabidopsis (Arabidopsis thaliana). Up to $9.73 \pm 2.73 \mu \mathrm{g}$ $\mathrm{OD}_{730}{ }^{-1} \mathrm{~L}^{-1}$ of fatty alcohol was obtained in the Synechocystis strain expressing the jojoba FAR gene [15]. The yield of fatty alcohol in cyanobacteria was then increased to approximately $761 \mathrm{ug} / \mathrm{g}$ dry mass by blocking the glycogen synthesis and poly-3-hydroxybutyrate (PHB) synthesis as well as by over-expressing the FAR genes [39]. However, compared with E. coli and yeast, the construction of fatty alcohol biosynthetic pathway in cyanobacteria is still in its infancy. Thus, more genetic engineering work is needed to improve the cyanobacterial fatty alcohol production, such as the introduction of the novel FAR genes into cyanobacterial hosts or the disruption of the competitive pathway.

In this study, the maqu_2220 gene was heterologously expressed in Synechocystis for the production of fatty alcohols. In order to improve the fatty alcohol synthesis, the acyl-ACP reductase (encoded by sll0209) and aldehydedeformylating oxygenase (encoded by sll0208) in the fatty alkane biosynthesis pathway were deleted in the maqu_2220-expressing Synechocystis strain (Figure 1B). These approaches resulted in a significant increase of cyanobacterial fatty alcohol production, which reached up to $2.87 \mathrm{mg} / \mathrm{g}$ dry weight.

\section{Results and discussion}

Production of fatty alcohols by heterologously expressing the maqu_2220 gene in Synechocystis

The maqu_2220 gene from $M$. aquaeolei VT8 was expressed under the control of the promoter $\mathrm{P}_{\text {petE }}$ (Nucleotide sequences listed in the Additional file 3); the Omega cassette [40] which harbors a spectinomycin resistance gene was used as a selection marker (Figure 2A). The mutant strain Syn-FQ52 was obtained by transformation of Synechocystis with the plasmid pFQ52. The expressing cassette was integrated into the slr0168 locus of the genome by double homologous recombination. The integration of the expressing cassette and the complete segregation of the mutant strain were confirmed by PCR analysis (see Additional file 4: Figure S2). The most abundant fatty alcohols in Syn-FQ52 are hexadecanol and octadecanol, which are the same as those in the strain expressing the jojoba FAR [15]. Syn-FQ52 was able to produce fatty alcohols with a yield of $0.21 \pm 0.03 \mathrm{mg} / \mathrm{g}$ Dry Weight (DW), $0.29 \pm 0.04 \mathrm{mg} / \mathrm{g} \mathrm{DW}$ and $0.39 \pm 0.06 \mathrm{mg} / \mathrm{g}$ DW in total after culturing for 140, 274, and 384 hours, respectively. No fatty alcohol was detected in the wild-type Synechocystis strain (Figures 3 and 4B).

The bacterial FAR Maqu_2220 was shown to have broad substrate activity towards fatty acyl-CoAs and fatty aldehydes with different carbon chain lengths and unsaturation $[35,36]$. However, no unsaturated fatty alcohols were detected in our maqu_2220-expressing strains of Synechocystis. It suggests that the profile of fatty alcohols not only depends on the substrate specificity of FARs but can also be strongly influenced by the host organism.

Deletion of the aldehyde-deformylating oxygenase gene from the hydrocarbon biosynthesis pathway affected the alka(e)ne and fatty acids production

The ADO gene sll0208 in both the wild-type Synechocystis and Syn-FQ52 was inactivated by inserting a kanamycin resistance gene in the middle of the sll0208 gene (Figure 2B), generating Syn-D08 and Syn-FQ52D08, respectively. The gene replacement was confirmed by PCR assay. Only a single $3.3 \mathrm{~kb}$-band was amplified from the genomic DNA of the sll0208 mutant strain. The band size is not the same as that in the wild-type control (see Additional file 4: Figure S2). This indicates that the sll0208 mutant was completely segregated and the sll0208 gene was completely disrupted.

Syn-FQ52 showed similar fatty alka(e)ne and free fatty acid accumulations as the wild-type Synechocystis (Additional file 5: Figure S3 and Figure 4C). Fatty alka (e)nes took a large proportion of all the fatty acid derivatives in Synechocystis (Additional file 5: Figure S3C and D). Heptadecane and heptadecene were the most abundant hydrocarbons in the wild-type Synechocystis. By contrast, there were no hydrocarbons detected in Syn-D08 and Syn-FQ52D08, indicating that the alka(e) ne biosynthesis pathway was completely inactivated by the disruption of sllo208.

As the hydrocarbon synthesis was blocked, a large amount of fatty aldehydes was supposed to accumulate in the sll0208 deletion strains. However, no fatty aldehyde was detected (Additional file 6: Figure S4) in Syn-D08 and Syn-FQ52D08 based on the extraction and Gas chromatography-mass spectrometry (GC-MS) method in this study. As controls, the pure fatty aldehydes proved to be quite stable under the same extraction and analysis procedures (Additional file 7: Figure S5). 


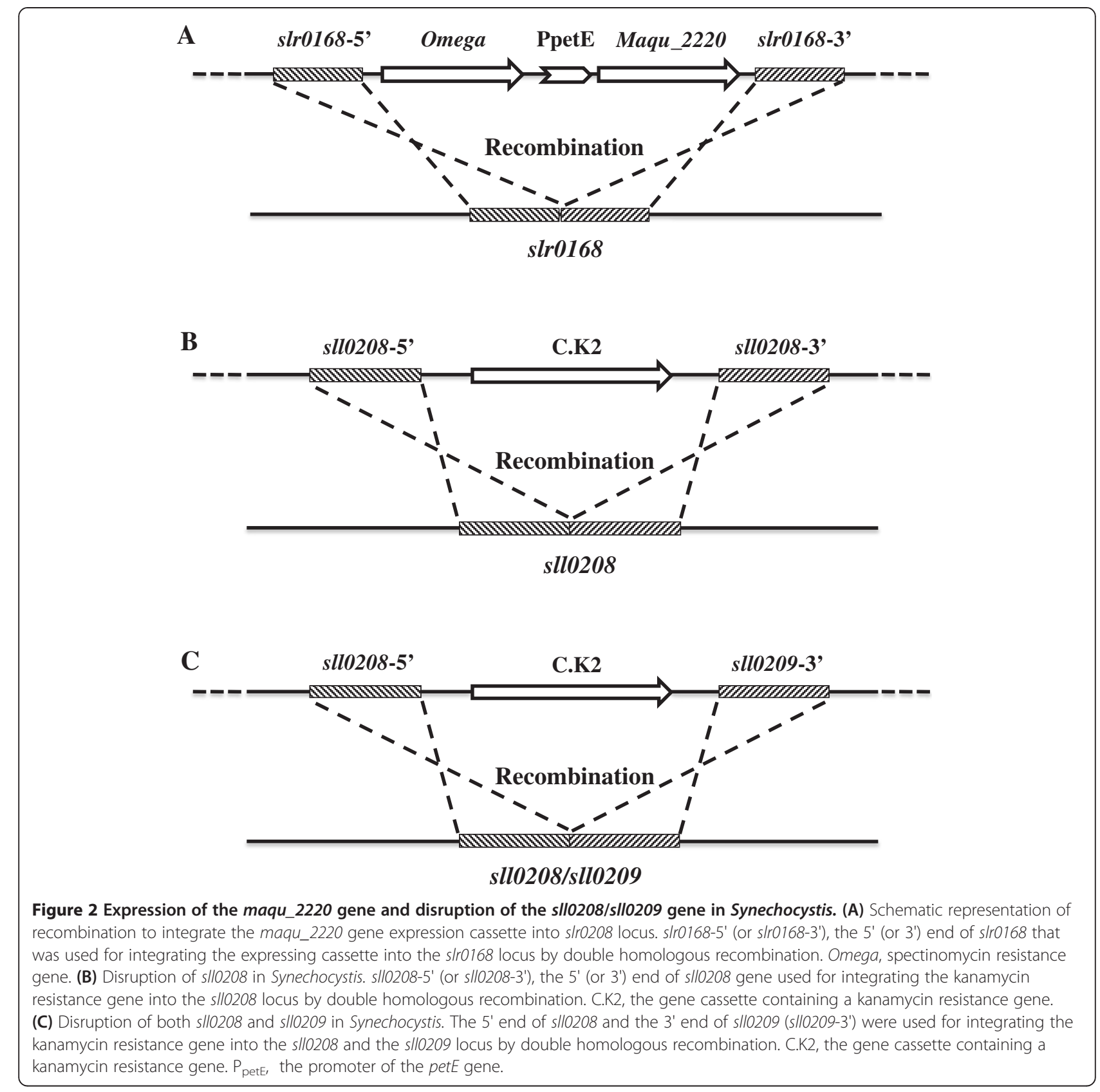

One possibility is that the generated fatty aldehydes were oxidized by the newly discovered aldehyde dehydrogenase in cyanobacteria $[26,41]$. Kaiser et al. detected fatty aldehyde in S. elongatus PCC 7942 with over-expression of the fatty acyl-ACP reductase (encoded by orf1594) and deletion of the aldehyde dehydrogenase (encoded by orf0489) [41]. The ortholog of Orf0489 in Synechocystis, Slr0091, had also been proved to possess the activity of converting fatty aldehyde to fatty acids [26]. Thus, there is a chance that the fatty aldehydes accumulated in Syn-D08 and Syn-FQ52D08 were efficiently converted to fatty acids by the aldehyde dehydrogenase. In accordance with this, the total free fatty acids in Syn-D08 and Syn-FQ52D08 increased dramatically compared to WT strain (Figure 4C). Further, as an intermediate of the fatty acid pathway, fatty aldehydes are probably toxic for cyanobacteria, thus cyanobacteria may only possess a low level of intracellular fatty aldehyde pools.

The enhanced production of fatty alcohols in Synechocystis by the deletion of the sll0208 gene By inactivation of the sll0208 gene, no hydrocarbon was detected and the metabolic flux was redirected to the synthesis of fatty alcohols. Figure 4B shows the yield of 


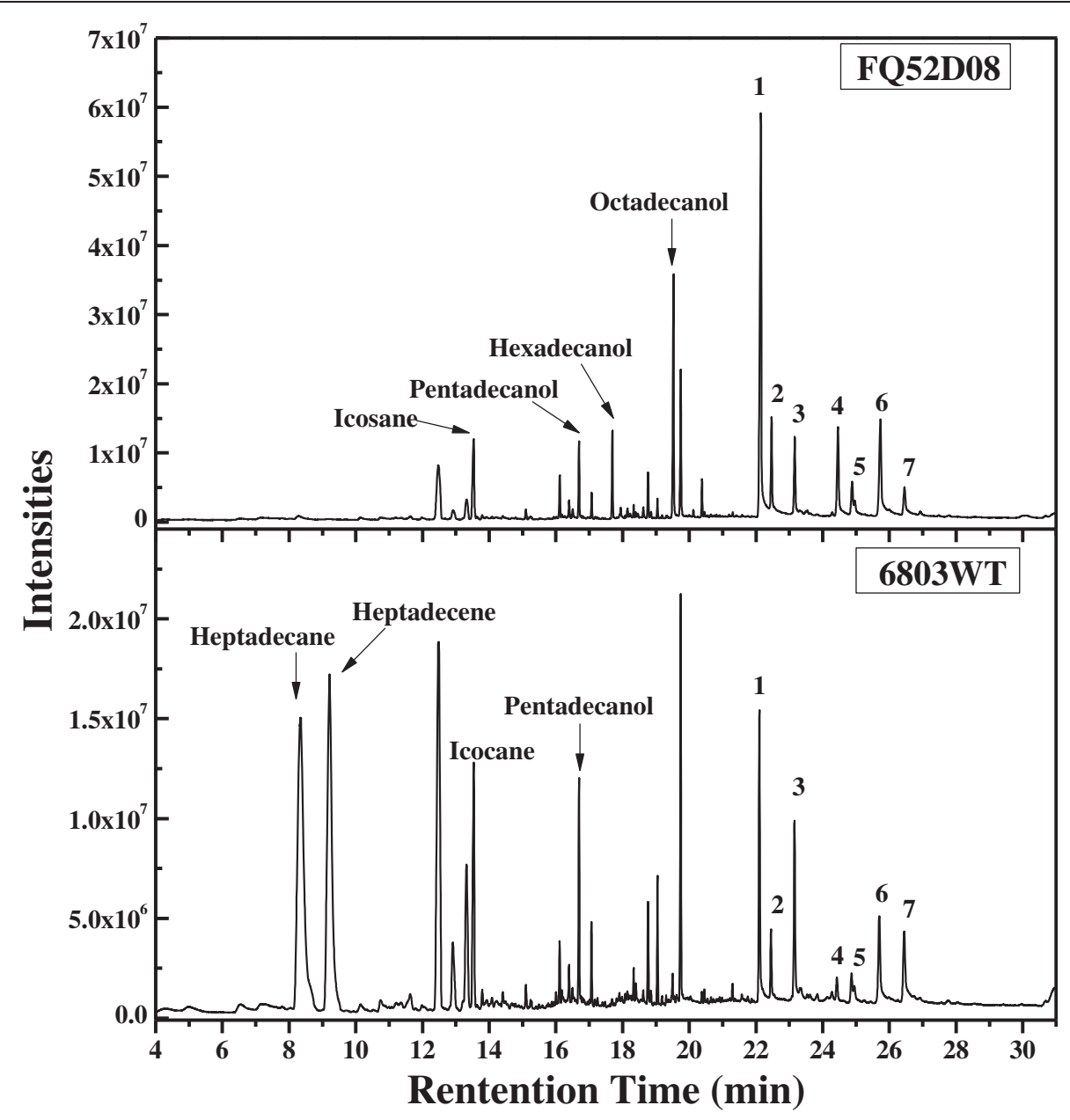

Figure 3 GC-MS analysis of alkanes, fatty alcohols and free fatty acids in wild-type Synechocystis and Syn-FQ52D08. Peaks: (1) palmitic acid; (2) palmitoleic acid; (3) heptadecanoic acid; (4) stearic acid; (5) oleic acid; (6) linoleic acid; (7) linolenic acid. Eicosane, pentadecanol, and heptadecanoic acid were used as internal standards for the analysis of alkanes, fatty alcohols, and free fatty acids respectively. 6803WT: the wild-type strain of Synechocystis; Syn-FQ52D08: the strain with deletion of sll0208 and expression of maqu_2220.

fatty alcohol in the mutant and wild type strains of Synechocystis culturing for 140, 274 and 384 hours. The yield of fatty alcohols in Syn-FQ52D08 showed a significant increase (2.8, 3.6, and 2.3-fold of that in Syn-FQ52 at 140,274 , and 384 hours, respectively), reaching up to $0.6 \pm 0.06,1.03 \pm 0.21$, and $0.91 \pm 0.05 \mathrm{mg} / \mathrm{g} \mathrm{DW}$ (Figure 4B). The fatty alcohol productivity reported here had a significant improvement compared to that in Syn-XT14 $\left(9.73 \pm 2.73 \mu \mathrm{g} \mathrm{OD}_{730}{ }^{-1} \mathrm{~L}^{-1}\right)$ as previously published by our lab [15].

The free fatty acids (FFA) content was also increased in Syn-FQ52D08 (Figure 4C), which was about 3.4 times, 4 times and 7 times higher compared to that of Syn-FQ52 respectively. In contrast, the FFA content in Syn-D08 was only slightly increased compared to that of the wild-type Synechocystis (Figure 4C). More obviously, the total fatty acyl chains (fatty alcohol, hydrocarbon, and free fatty acid) in Syn-FQ52D08 were much more than that in Syn-FQ52 and Syn-D08 (Additional file 5: Figure S3D). It suggests that more carbon flux could be driven into the fatty acid biosynthesis pathway by expressing the Maqu_2220 and disruption of the sll0208 gene in Syn-FQ52D08.

\section{A further increase in fatty alcohol production by double knock-out of sll0208 and sll0209}

Even though Maqu_2220 from M. aquaeolei VT8 has been proved to catalyze both the reduction of fatty acylCoA (or acyl-ACP) to fatty alcohols and the reduction of fatty aldehydes to fatty alcohols [35,36], it was unclear which pathway (Figure 1B) contributes to the increase of fatty alcohols in the mutant strain Syn-FQ52D08. The mutant strain Syn-FQ52D0809 was thereby constructed by inactivation of both the sll0208 and sll0209 gene in Syn-FQ52 (Figure 2C).

Figure 4A compared the growth of the mutant and the wild type strains of Synechocystis. It can be found that 

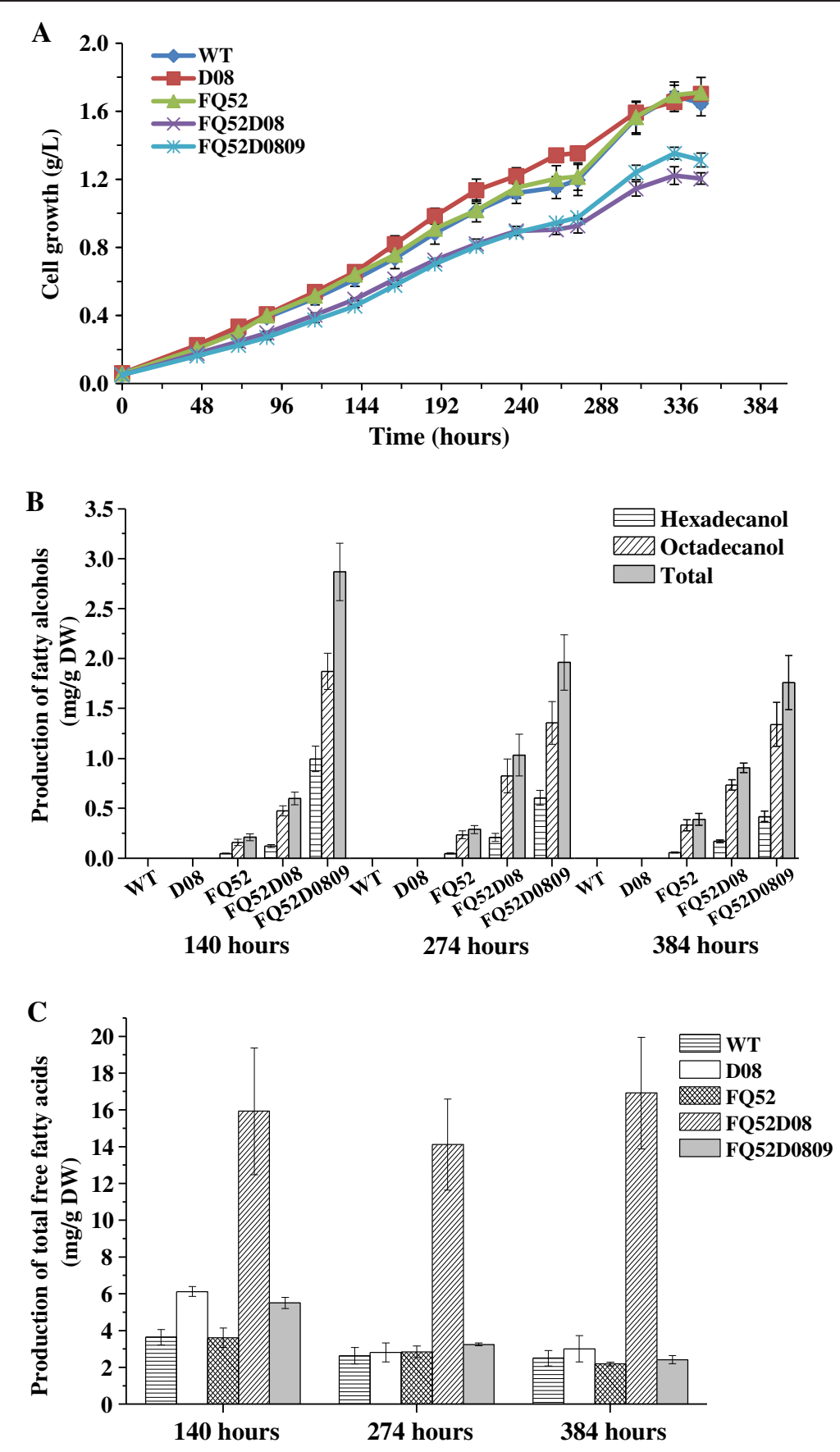

Figure 4 Contents and composition of fatty acid derivatives in the mutant and the wild-type Synechocystis. (A) Growth curves of WT, Syn-D08, Syn-FQ52, Syn-FQ52D08, and Syn-FQ52D0809; (B) Fatty alcohol production in the Synechocystis mutant strains; (C) Total free fatty acid production in Synechocystis WT and mutant strains. Error bars indicate standard deviation $(n=3)$. WT: the wild-type strain of Synechocystis; SynD08: the strain with deletion of s/l0208; Syn-FQ52: the strain with expression of maqu_2220; Syn-FQ52D08: the strain with deletion of s/l0208 and expression of maqu_2220; Syn-FQ52D0809: the strain with expression of maqu_2220 and double knockout of sll0208 and sll0209.

the Syn-D08 and Syn-FQ52 showed a similar growth trend as the wild type strain, while the mutant Syn-FQ52D08 and Syn-FQ52D0809 exhibited the slightly lower growth rate. Figure 4B showed a further increase in fatty alcohol production by deletion of both the sll0208 and the sll0209 gene. The fatty alcohol yield in Syn-FQ52D0809 was $2.87 \pm$ $0.29,1.96 \pm 0.28$, and $1.76 \pm 0.27 \mathrm{mg} / \mathrm{g}$ DW after 140, 274 and 384 hours' cultivation, respectively $(5,1.9$, and 1.93-fold of the fatty alcohol yield in Syn-FQ52D08, respectively). Except for inactivation of the fatty alka(e)ne synthesis, the 
deletion of sll0209 would block the fatty aldehydes synthesis as well as its downstream fatty acids synthesis that is catalyzed by the fatty aldehyde dehydrogenase. The enhanced production of fatty alcohols could be attributed to the increase of the fatty acyl-CoA (or acyl-ACP) pool in Synechocystis by the inactivation of sll0208 and sll0209.

Syn-FQ52, Syn-FQ52D08, and Syn-FQ52D0809 showed different trends of the fatty alcohol productivity over the three time points. The yield of fatty alcohols of Syn-FQ52 showed a continuous increase during the 16 days of culturing, while the fatty alcohol productivity in Syn-FQ52D0809 decreased as the cell growth increased. Syn-FQ52D08 showed a different trend in fatty alcohol productivity. It was $0.6 \pm 0.06 \mathrm{mg} / \mathrm{g} \mathrm{DW}$ at 140 hours and subsequently reached a maximum at 274 hours $(1.03 \pm 0.21 \mathrm{mg} / \mathrm{g} \mathrm{DW})$, followed by a slight decrease at 384 hours $(0.91 \pm 0.05 \mathrm{mg} /$ $\mathrm{g} D W)$. This indicates that fatty alcohol synthesis is related with the genotype of the strains and their growth phases. Furthermore, we also found that the newly transformed strains provided better fatty alcohol production. These results indicate that unknown mechanisms may exist for the regulation of the heterologous fatty alcohol synthesis in cyanobacteria.

The proportion of fatty acids, fatty alkane, and fatty alcohols in all strains were compared in Additional file 5: Figure S3. In strain Syn-FQ52D0809, the fatty alcohols comprised approximately $40 \%$ of the fatty acyl chains (Additional file 5: Figure S3C). In another aspect, however, it was found that the deletion of sll0208 can dramatically raise the percentage of fatty acids. For instance, Syn-D08 mainly produced approximately $99 \%$ fatty acids, while Syn-FQ52D08 produced approximately 95\% fatty acids and $5 \%$ fatty alcohols. These strains may play important roles in the production of fatty acid-based biofuels in cyanobacteria in future.

Compared to the heterotrophic bacteria such as E. coli and yeast, the current fatty alcohol productivity by cyanobacteria is still too low. Several additional approaches can be applied to improve Synechocystis engineering efficiency. First, heterologous gene expression can be improved by optimizing synonymous codon usage to better match the cyanobacterial host [42]. Second, the fatty alcohol biosynthesis can be enhanced by introducing more metabolic flux. Blocking the competing pathway of the PHB synthesis and the glycogen synthesis can drive the metabolic flux towards fatty alcohol synthesis [39]. Atsumi et al. showed overexpression of ribulose 1,5-bisphosphate carboxylase/ oxygenase (RuBisCO) improves cyanobacterial isobutyraldehyde and isobutanol alcohol biosynthesis [43]. It would also be beneficial to the synthesis of long chain fatty alcohols. Third, the cultivation condition may also be improved by using higher light intensity (in the late growth phase) and higher $\mathrm{CO}_{2}$ concentration [15,43]. In a large scale and higher cell-density culture, light and $\mathrm{CO}_{2}$ can be limiting factors for cyanobacterial biosynthesis. Thereby, mixotrophic conditions may be an alternative solution. For example, Synechocystis can uptake acetate for acetylCoA synthesis [44] and improve product yield, and thus cheap carbon substrate can be employed for photobiorefinery.

\section{Conclusions}

In this study, the biosynthesis of fatty alcohols was achieved by expressing a fatty acyl-CoA reductase gene maqu_2220 from M. aquaeolei VT8. The double knockout of the native sll0208 and sll0209 involved in the alkane biosynthesis pathway in Synechocystis improved the production of fatty alcohols. The results indicate that the deletion of the competing pathways for fatty alcohol biosynthesis could significantly improve the product yield.

The diverse fatty acyl-CoA (fatty acyl-ACP) reductases (Additional file 3: Table S1, Additional file 1: Figure S1) display a huge potential to molecularly modulate fatty alcohol product with the desired chain length and saturation degree, and provides feasibility to achieve the desired production of fatty alcohol-based biofuels and biochemicals. Further modifications of the metabolic network toward fatty acid biosynthesis are still needed for the significant improvement and application of photosynthetic fatty alcohol production in cyanobacteria.

\section{Methods}

\section{Plasmid and strain construction}

The prokaryotic fatty acyl-CoA reductase gene maqu_2220 from M. aquaeolei VT8 (DSM 11845, purchased from Deutsche Sammlung von Mikroorganismen und Zellkulturen) was amplified by PCR using the primer pair faldr-1 and faldr-2 (Additional file 8: Table S2). It was sub-cloned into the cloning vector pMD18-T (Takara Co., Ltd, Kyoto, Japan) resulting in pXT71. The maqu_2220 fragment in pXT71 was digested with NdeI/XhoI (Takara Co., Ltd, Kyoto, Japan) and ligated to the same site of pXT37b [15] generating the expression vector $\mathrm{pFQ} 52$. Then $\mathrm{pFQ} 52$ was transformed into the wild-type strain of Synechocystis. Transformation of Synechocystis was performed as described previously [15].

The plasmid pD0208 was constructed to disrupt the sll0208 gene via homologous recombination in Synechocystis. A $2.4 \mathrm{~kb}$ of DNA fragment, which includes the open reading frame (ORF) and the upstream and downstream flanking regions of sllo208, was PCR-amplified from the genomic DNA of Synechocystis using primers D0208-F and D0208-R. This fragment was cloned into pGEM-T easy vector (Promega, USA) generating pGEMT-D0208. The plasmid pGEMT-D0208 was digested with Hind III (Takara Co., Ltd, Kyoto, Japan), blunted by T4 DNA polymerase (Thermo Scientific, USA) and ligated with the 
kanamycin resistance gene cassette C.K2 [45], which was amplified from the pRL446 plasmid [45] using primers C. K2-NF and C.K2-NR (Additional file 8: Table S2), generating the plasmid pD0208. The plasmid pLY76 was constructed to disrupt both the sll0208 and sll0209 gene in Synechocystis. Two $1.1 \mathrm{~kb}$ DNA fragments that included the upstream flanking region of sll0208 and the downstream flanking region of sll0209 were amplified using primer pairs D0809-1/D0809-2 and D0809-3/D0809-4 (Additional file 8: Table S2), respectively. The two DNA fragments containing a 33-bp overlapping region were used as templates to amplify a $2.3 \mathrm{~kb}$ DNA fragment. The fused fragment was sub-cloned into pMD18-T generating pLY75. The C.K2 cassette from the pRL446 plasmid [45] was sub-cloned into the blunted Bam HI site of pLY75 to generate the plasmid pLY76. The plasmid pD0208 and pLY76 were transformed into Synechocystis for homologous integration. The single knockout of the sll0208 gene and the double knockout of sll0208 and sll0209 were verified by PCR.

\section{Synechocystis culture conditions}

All Synechocystis strains were cultured at $30^{\circ} \mathrm{C}$ in $500 \mathrm{~mL}$ flasks containing $450 \mathrm{~mL}$ BG11 medium bubbled with air under constant fluorescent light at an intensity of 30 to $50 \mu \mathrm{E} \mathrm{m}^{-2} \mathrm{~s}^{-1}$. Cells were grown for 16 days in total and were sampled at 140,274, and 384 hours for the measuring of fatty alcohols. Spectinomycin $\left(20 \mu \mathrm{g} \mathrm{mL} \mathrm{m}^{-1}\right)$ and/or kanamycin $\left(20 \mu \mathrm{g} \mathrm{m}^{-1}\right)$ (Solarbio, Beijing, China) was added to the medium when needed. The correlation between the cell dry weight (DW) and optical density (OD) of the Synechocystis cells was confirmed by measuring both the $\mathrm{OD}_{730}$ and the cell Dry Weight (See Additional file 9: Figure S6 and Additional file 10: Table S3).

\section{Lipids extraction and GC-MS analysis}

Synechocystis cells from $150 \mathrm{~mL}$ of culture were harvested by centrifugation, re-suspended in $10 \mathrm{~mL}$ of TE buffer (10 mM Tris-HCl, 1 mM EDTA, pH 8.0) and then lysed by sonication. The lysate was extracted for 30 minutes at room temperature with $10 \mathrm{~mL}$ chloroform-methanol (v/v, 2:1). Prior to extraction, $30 \mu \mathrm{g}$ 1-pentadecanol, $50 \mu \mathrm{g}$ eicosane, and $100 \mu \mathrm{g}$ heptadecanoic acid (Sigma Aldrich, USA) were added into the cell lysate as internal standards (Figure 4). The organic phase was separated by centrifugation and evaporated to dryness under nitrogen at $55^{\circ} \mathrm{C}$. The dryness was dissolved in $1 \mathrm{~mL}$ of $\mathrm{n}$-hexane and $1-\mu \mathrm{L}$ aliquot was analyzed by GC-MS using an Agilent 7890A system equipped with a HP-INNOWax $(30 \mathrm{~m} \times 250 \mu \mathrm{m} \times$ $0.25 \mu \mathrm{m}$, Agilent, USA). Helium (constant flow $1 \mathrm{~mL}$ $\min ^{-1}$ ) was used as carrier gas. The temperature of the injector was $250^{\circ} \mathrm{C}$. The following temperature program was applied: $100^{\circ} \mathrm{C}$ for 1 minute, increase of $5^{\circ} \mathrm{C} \mathrm{min}^{-1}$ to $150^{\circ} \mathrm{C}$ and then $10^{\circ} \mathrm{C} \mathrm{min}{ }^{-1}$ to $250^{\circ} \mathrm{C}$, hold at $250^{\circ} \mathrm{C}$ for 15 minutes. The internal standards were used to determine the product yield, which was the mean of three independent experiments [15].

\section{Additional files}

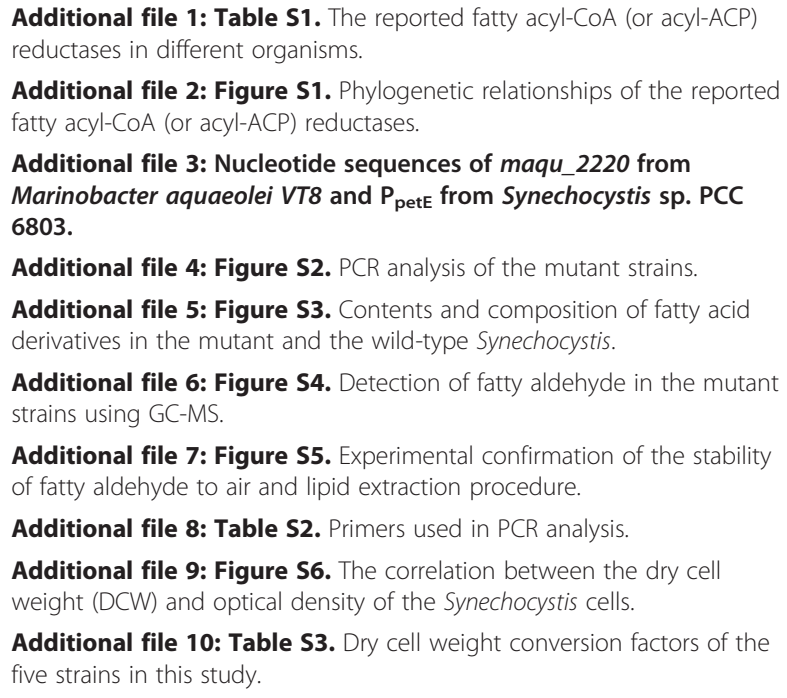

Additional file 2: Figure S1. Phylogenetic relationships of the reported fatty acyl-CoA (or acyl-ACP) reductases.

Additional file 3: Nucleotide sequences of maqu_2220 from Marinobacter aquaeolei VT8 and $\mathrm{P}_{\text {petE }}$ from Synechocystis sp. PCC 6803.

Additional file 4: Figure S2. $P C R$ analysis of the mutant strains.

Additional file 5: Figure S3. Contents and composition of fatty acid derivatives in the mutant and the wild-type Synechocystis.

Additional file 6: Figure S4. Detection of fatty aldehyde in the mutant strains using GC-MS.

Additional file 7: Figure S5. Experimental confirmation of the stability of fatty aldehyde to air and lipid extraction procedure.

Additional file 8: Table S2. Primers used in PCR analysis.

Additional file 9: Figure S6. The correlation between the dry cell weight (DCW) and optical density of the Synechocystis cells.

Additional file 10: Table S3. Dry cell weight conversion factors of the five strains in this study.

\section{Abbreviations}

AAR: acyl-ACP reductase; ACP: Acyl carrier protein; ADO: Aldehyde-deformylating oxygenase; DW: dry weight; FAR: Fatty acyl-CoA reductase; FFA: Free fatty acid; GC-MS: Gas chromatography-mass spectrometry. OD, optical density; PHB: poly-3-hydroxybutyrate.

\section{Competing interests}

The authors declare that they have no competing interests.

\section{Authors' contributions}

LY and FQ carried out the construction and cultivation of Synechocystis sp. PCC 6803 mutant strains, extraction and analysis of free fatty acids, fatty alka (e)nes and fatty alcohols. LY, FQ and XL designed the experiments. LY and $\mathrm{XL}$ drafted the manuscript. $\mathrm{XT}$ and $\mathrm{FQ}$ participated in the design of the study and revised the manuscript. $\mathrm{XL}$ conceived of the study. All authors read and approved the final manuscript.

\section{Acknowledgements}

This work was supported by the '100-Talent Program of the Chinese Academy of Sciences' foundation (grant number: O91001110A).

\section{Author details}

${ }^{1}$ Key Laboratory of Biofuels, Shandong Provincial Key Laboratory of Energy Genetics, Qingdao Institute of Bioenergy and Bioprocess Technology, Chinese Academy of Sciences, No. 189 Songling Road, Qingdao 266101, China. ${ }^{2}$ University of Chinese Academy of Sciences, No. 19 jia, Yuquan Street, Shijingshan District, Beijing 100049, China.

Received: 4 November 2013 Accepted: 4 June 2014

Published: 18 June 2014

\section{References}

1. Dellomonaco C, Fava F, Gonzalez R: The path to next generation biofuels: successes and challenges in the era of synthetic biology. Microb Cell Fact 2010, 9:3.

2. Lee SK, Chou H, Ham TS, Lee TS, Keasling JD: Metabolic engineering of microorganisms for biofuels production: from bugs to synthetic biology to fuels. Curr Opin Biotech 2008, 19:556-563. 
3. Rude MA, Schirmer A: New microbial fuels: a biotech perspective. Curr Opin Microbiol 2009, 12:274-281.

4. Fortman JL, Chhabra S, Mukhopadhyay A, Chou H, Lee TS, Steen E, Keasling JD: Biofuel alternatives to ethanol: pumping the microbial well. Trends Biotechnol 2008, 26:375-381

5. Zhang FZ, Rodriguez S, Keasling JD: Metabolic engineering of microbia pathways for advanced biofuels production. Curr Opin Biotech 2011, 22:775-783.

6. Lu X: A perspective: photosynthetic production of fatty acid-based biofuels in genetically engineered cyanobacteria. Biotechnol Adv 2010, 28:742-746

7. Parmar A, Singh NK, Pandey A, Gnansounou E, Madamwar D: Cyanobacteria and microalgae: a positive prospect for biofuels. Bioresour Technol 2011, 102:10163-10172.

8. Shih PM, Wu DY, Latifi A, Axen SD, Fewer DP, Talla E, Calteau A, Cai F, de Marsac NT, Rippka R, Herdman M, Sivonen K, Coursin T, Laurent T, Goodwin L, Nolan M, Davenport KW, Han CS, Rubin EM, Eisen JA, Woyke T, Gugger M, Kerfeld CA: Improving the coverage of the cyanobacterial phylum using diversity-driven genome sequencing. Proc Natl Acad Sci U S A 2013, 110:1053-1058.

9. Angermayr SA, Hellingwerf KJ, Lindblad P, de Mattos MJT: Energy biotechnology with cyanobacteria. Curr Opin Biotech 2009, 20:257-263.

10. Ducat DC, Way JC, Silver PA: Engineering cyanobacteria to generate high-value products. Trends Biotechnol 2011, 29:95-103.

11. Deng MD, Coleman JR: Ethanol synthesis by genetic engineering in cyanobacteria. Appl Environ Microbiol 1999, 65:523-528.

12. Lindberg P, Park S, Melis A: Engineering a platform for photosynthetic isoprene production in cyanobacteria, using Synechocystis as the model organism. Metab Eng 2010, 12:70-79.

13. Atsumi S, Higashide W, Liao JC: Direct photosynthetic recycling of carbon dioxide to isobutyraldehyde. Nat Biotechnol 2009, 27:1177-U1142.

14. Shestakov SV, Mikheeva LE: Genetic control of hydrogen metabolism in cyanobacteria. Russ J Genet 2006, 42:1272-1284.

15. Tan XM, Yao L, Gao QQ, Wang WH, Qi FX, Lu XF: Photosynthesis driven conversion of carbon dioxide to fatty alcohols and hydrocarbons in cyanobacteria. Metab Eng 2011, 13:169-176.

16. Lan E, Liao J: Metabolic engineering of cyanobacteria for 1-butanol production from carbon dioxide. Metab Eng 2011, 13:353-363.

17. Liu X, Sheng J, Curtiss $R$ 3rd: Fatty acid production in genetically modified cyanobacteria. Proc Natl Acad Sci U S A 2011, 108:6899-6904.

18. Niederholtmeyer $\mathrm{H}$, Wolfstadter BT, Savage DF, Silver PA, Way JC: Engineering cyanobacteria to synthesize and export hydrophilic products. Appl Environ Microbiol 2010, 76:6023-6023.

19. Dexter J, Fu PC: Metabolic engineering of cyanobacteria for ethanol production. Energ Environ Sci 2009, 2:857-864.

20. Reinsvold RE, Jinkerson RE, Radakovits R, Posewitz MC, Basu C: The production of the sesquiterpene beta-caryophyllene in a transgenic strain of the cyanobacterium Synechocystis. J Plant Physiol 2011, $168: 848-852$.

21. Ducat DC, Avelar-Rivas JA, Way JC, Silver PA: Rerouting carbon flux to enhance photosynthetic productivity. Appl Environ Microbiol 2012, 78:2660-2668

22. Kaczmarzyk D, Fulda M: Fatty acid activation in cyanobacteria mediated by acyl-acyl carrier protein synthetase enables fatty acid recycling. Plant Physiol 2010, 152:1598-1610.

23. Gao Q, Wang W, Zhao H, Lu X: Effects of fatty acid activation on photosynthetic production of fatty acid-based biofuels in Synechocystis sp. PCC6803. Biotechnol Biofuels 2012, 5:17.

24. Schirmer A, Rude MA, Li X, Popova E, del Cardayre SB: Microbial Biosynthesis of Alkanes. Science 2010, 329:559-562.

25. Danika T, Bjorn V, Annegret W, Salim A, Wolfgang RH: Microevolution in Cyanobacteria: Re-sequencing a Motile Substrain of Synechocystis sp PCC 6803. DNA Res 2012, 19:435-448.

26. Trautmann D, Beyer P, Al-Babili S: The ORF slr0091 of Synechocystis sp. PCC6803 encodes a high-light induced aldehyde dehydrogenase converting apocarotenals and alkanals. FEBS J 2013, 280:3685-3696.

27. Metz JG, Pollard MR, Anderson L, Hayes TR, Lassner MW: Purification of a jojoba embryo fatty acyl-coenzyme $A$ reductase and expression of its CDNA in high erucic acid rapeseed. Plant Physiol 2000, 122:635-644.
28. Rowland $\mathrm{O}$, Zheng HQ, Hepworth SR, Lam P, Jetter R, Kunst L: CER4 encodes an alcohol-forming fatty acyl-coenzyme $A$ reductase involved in cuticular wax production in Arabidopsis. Plant Physiol 2006, 142:866-877.

29. Doan TTP, Carlsson AS, Hamberg M, Bulow L, Stymne S, Olsson P: Functional expression of five Arabidopsis fatty acyl-CoA reductase genes in Escherichia coli. J Plant Physiol 2008, 166:787-796.

30. Cheng JB, Russell DW: Mammalian wax biosynthesis - I. Identification of two fatty acyl-coenzyme A reductases with different substrate specificities and tissue distributions. J Biol Chem 2004, 279:37789-37797.

31. Vioque J, Kolattukudy PE: Resolution and purification of an aldehyde-generating and an alcohol-generating fatty acyl-CoA reductase from pea leaves (Pisum sativum $L$ ). Arch Biochem Biophys 1997, 340:64-72.

32. Teerawanichpan P, Qiu X: Fatty Acyl-CoA Reductase and Wax Synthase from Euglena gracilis in the Biosynthesis of Medium-Chain Wax Esters. Lipids 2010, 45:263-273.

33. Willis RM, Wahlen BD, Seefeldt LC, Barney BM: Characterization of a fatty acyl-CoA reductase from Marinobacter aquaeolei VT8: a bacterial enzyme catalyzing the reduction of fatty acyl-CoA to fatty alcohol. Biochemistry 2011, 50:10550-10558.

34. Reiser S, Somerville C: Isolation of mutants of Acinetobacter calcoaceticus deficient in wax ester synthesis and complementation of one mutation with a gene encoding a fatty acyl coenzyme a reductase. J Bacterio/ 1997, 179:2969-2975

35. Wahlen BD, Oswald WS, Seefeldt LC, Barney BM: Purification, characterization, and potential bacterial wax production role of an NADPH-dependent fatty aldehyde reductase from Marinobacter aquaeolei VT8. Appl Environ Microbiol 2009, 75:2758-2764.

36. Hofvander P, Doan TTP, Hamberg M: A prokaryotic acyl-CoA reductase performing reduction of fatty acyl-CoA to fatty alcohol. FEBS Lett 2011 585:3538-3543.

37. Liu A, Tan X, Yao L, Lu X: Fatty alcohol production in engineered E. coli expressing Marinobacter fatty acyl-CoA reductases. Appl Microbiol Biotechnol 2013, 97:7061-7071.

38. Runguphan W, Keasling JD: Metabolic engineering of Saccharomyces cerevisiae for production of fatty acid-derived biofuels and chemicals. Metab Eng 2014, 21:103-113.

39. Qi F, Yao L, Tan X, Lu X: Construction, characterization and application of molecular tools for metabolic engineering of Synechocystis sp. Biotechnol Lett 2013, 35:1655-1661.

40. Prentki $P$, Binda A, Epstein A: Plasmid vectors for selecting IS1-promoted deletions in cloned DNA: sequence analysis of the omega interposon. Gene 1991, 103:17-23.

41. Kaiser BK, Carleton M, Hickman JW, Miller C, Lawson D, Budde M, Warrener P, Paredes A, Mullapudi S, Navarro P, Cross F, Roberts JM: Fatty aldehydes in cyanobacteria are a metabolically flexible precursor for a diversity of biofuel products. PLoS One 2013, 8:e58307.

42. Varman AM, Yu Y, You L, Tang YJ: Photoautotrophic production of D-lactic acid in an engineered cyanobacterium. Microb Cell Fact 2013, 12:117.

43. Atsumi S, Higashide W, Liao JC: Direct photosynthetic recycling of carbon dioxide to isobutyraldehyde. Nat Biotech 2009, 27:1177-1180.

44. Wu G, Bao T, Shen Z, Wu Q: Sodium acetate stimulates PHB biosynthesis in Synechocystis sp. PCC 6803. Tsinghua Sci Technol 2002, 7:435-438.

45. Xu XD, Yin CT, Li WZ, Du Y, Kong RQ: Identification of a gene, ccr-1 (s/11242), required for chill-light tolerance and growth at 15 degrees $C$ in Synechocystis sp PCC 6803. Microbiol-Sgm 2007, 153:1261-1267.

doi:10.1186/1754-6834-7-94

Cite this article as: Yao et al:: Improved production of fatty alcohols in cyanobacteria by metabolic engineering. Biotechnology for Biofuels 2014 7:94. 